\title{
Hepatitis C virus treatment advances for thalassaemia patients
}

\author{
George Papatheodoridis
}

\author{
Professor in Medicine and Gastroenterology, Medical School of National and Kapodistrian \\ University of Athens, Director of Academic Department of Gastroenterology, Laiko General \\ Hospital, Athens, Greece
}

\begin{abstract}
Chronic infection with hepatitis $\mathrm{C}$ virus (HCV) is a major problem for thalassaemia patients, as blood transfusions before 1990 were associated with a high risk of HCV infection. Given the high prevalence of co-morbidities, thalassaemia patients are at an increased risk for dying from end-stage liver disease or hepatocellular carcinoma. HCV treatment in thalassaemia patients was challenging in the interferon-alfa (IFN) era not only due to its unfavourable safety and tolerability profile but due to necessary combined use of ribavirin (RBV) and the subsequent haemolysis and increased need for blood transfusions. The introduction of the current direct acting antivirals (DAAs), which can be used in IFNfree and RBV-free regimens, has dramatically improved the management of all HCV patients including those with thalassaemia. Currently, depending on HCV genotype and availability in each country, the main available DAAs combinations are the co-formulation of sofosbuvir with ledipasvir (nucleotide analogue NS5B polymerase inhibitor/NS5A inhibitor, one tablet of $400 / 90 \mathrm{mg}$ once daily), the co-formulation of paritaprevir boosted by ritonavir with ombitasvir (NS3/4 protease inhibitor/ritonavir/NS5A inhibitor, two tablets of $75 / 50 / 12.5 \mathrm{mg}$ once daily) perhaps with addition of dasabuvir (non-nucleos(t)ide analogue NS5B polymerase inhibitor, one tablet of $250 \mathrm{mg}$ twice daily), the co-formulation of grazoprevir with elbasvir (NS3/4 protease inhibitor/NS5A inhibitor, one tablet of $100 / 50 \mathrm{mg}$ once daily) and the co-formulation of sofosbuvir with velpatasvir (nucleotide analogue NS5B polymerase inhibitor/NS5A inhibitor, one tablet of 400/100 mg once daily). In 2017, the co-formulation of glecaprevir with
\end{abstract}

pibrentasvir (NS3/4 protease inhibitor/NS5A inhibitor, three tablets of 100/40 mg once daily) and the co-formulation of sofosbuvir with velpatasvir and voxilaprevir (nucleotide analogue NS5B polymerase inhibitor/NS5A inhibitor/ NS3/4 protease inhibitor, one tablet of 400/100/100 mg once daily) were also approved and started to be used in some countries.

According to all international current guidelines, thalassaemia patients do not represent a special group for the current HCV treatment and can be treated with the same indications and regimens used for patients without haemoglobinopathies. However, in countries which still prioritize the use of DAAs according to the severity of liver disease, thalassaemia patients are often excluded from such prioritization and have access to DAAs therapy regardless of their fibrosis severity. Moreover, all guidelines recommend that thalassaemia patients should be preferentially treated not only with IFN-free but RBV-free DAAs regimens too.

In a proper clinical trial, only a 12-week regimen of grazoprevir/elbasvir has been evaluated and proven to be highly efficacious and well tolerated among patients with inherited blood disorders and HCV genotype 1 or 4 infection. In addition, different DAAs regimens have been reported to be safe and effective for the treatment of $\mathrm{HCV}$ thalassaemia patiens in clinical practice. Given the availability of the current effective and safe DAAs and the frequent follow-up of thalassaemia patients in a few specific units, such patients could be a targeted population for "HCV micro-elimination" on the road towards the global HCV elimination in each country.

\author{
Correspondence: George Papatheodoridis, Medical School of National \\ and Kapodistrian University of Athens, Department of \\ Gastroenterology, Laiko General Hospital, Athens, Greece \\ This work is licensed under a Creative Commons Attribution 4.0 \\ License (by-nc 4.0). \\ CCopyright G. Papatheodoridis, 2018 \\ Licensee PAGEPress, Italy \\ Thalassemia Reports 2018; 8:7491 \\ doi:10.4081/thal.2018.7491
}

